\title{
Energy of a single electron in gaseous media
}

\author{
V.B. Shikin and S.S. Nazin \\ Institute of Solid State Physics, Chernogolovka, Moscow distr. 142432, Russia \\ E-mail: Shikin@issp.ac.ru
}

Received September 7, 2006

\begin{abstract}
The so-called «optical» approximation in the theory of electron energy spectrum in the presence of scattering centers with positive scattering length randomly distributed with the average density $n_{g}$ is considered for a number of inert gases. The average minimal energy $W$ of a single electron calculated beyond the optical approximation reveals a behavior qualitatively different from that of the same quantity $W_{0}$ derived within the optical approximation. Results of calculations are in qualitative agreement with experimental data available for the $W\left(n_{g}\right)$ dependence for different cryogenic gases.
\end{abstract}

PACS: 71.10.Ca Electron gas, Fermi gas;

71.10.-w Theories and models of many-electron systems.

Keywords: low-energy electron, excess electron, inert gases.

The interaction between charges and gaseous media (i.e. with randomly positioned scatterers with finite density) encompasses a large number of problems in low-temperature condensed matter and semiconductor physics. To be more specific, one can mention such wellknown phenomena as the development of charged bubbles and clusters in noble gases, including the influence of external fields, such as electric and magnetic, etc. There are also a number of effects which have not yet been given a consistent microscopic explanation, e.g. solvation (solvent density enhancement in the vicinity of charges caused by the interaction between neutral liquid atoms and the nonuniform electric field of a charged particle) and dissociation (decomposition of polar molecules in dielectric liquids). In the present paper we focus on the details of electron behavior in noble gases.

One of the basic characteristics of a low-energy electron in inert gases is its average minimum energy $W$ determining the behavior of the electron in such media (free motion at lengths much larger than interatomic distances or localization). In this context, the optical approximation (Ref. 1 and further publications [2-9] where this approach was developed; the list of references may be expanded)

$$
W_{0}=\frac{2 \pi \hbar^{2} a_{0}}{m} n_{g}
$$

is very useful. Here, $n_{g}$ is the average gas density, $m$ is the free-electron mass, and $a_{0}$ is the so-called scattering length of a slow electron on a given atom, which is closely related to the $s$-component of the electron scattering amplitude on the atom. Representation (1) is particularly valuable for solving inhomogeneous problems (such as bubble or cluster) where the local definition of the energy $W_{0}$ by Eq. (1) with coordinate-dependent density $n_{g}(r)$ has no alternative [10-13].

Equation (1) is certainly valid for the contact interaction $V_{0}\left(\mathbf{r}-\mathbf{R}_{i}\right)$ of the electron with atom:

$$
V_{0}\left(\mathbf{r}-\mathbf{R}_{i}\right)=\frac{2 \pi \hbar^{2} a_{0}}{m} \delta\left(\mathbf{r}-\mathbf{R}_{i}\right),
$$

where $\delta(x)$ is the Dirac delta-function. In fact, the actual interaction $V(r)$ is more complicated, and Eq. (2) only remains acceptable if the scattering length, which is an integral characteristic of $V(r)$, depends only slightly on $n_{g}$. The situation for inert gases seems to be acceptable, because $a_{0}$ is independent of $n_{g}$ as follows from data on the electron mobility in rarefied gases. For $\mathrm{He}, \mathrm{Ne}, \mathrm{Ar}, \mathrm{Kr}$, and $\mathrm{Xe}, a_{0} / a_{B}=+1.16,+0.45,-1.63,-3.8$, and -6.8 , respectively, where $a_{B}$ is the Bohr radius (see Refs. 14, 15, and the references therein). However, optical data indicate that the formation of an electron-helium bound state (negative helium ion has a binding energy of about $0.07 \mathrm{eV}$ [16-18]). Therefore, the scattering length in low-density gaseous helium that enters Eq. (2) is negative (we recall that an electron is pushed out liquid helium 
bulk with energy $W \sim 1 \mathrm{eV}[12,15,19-21]$; therefore, $a_{0}$ in Eq. (2) is positive in this case). Independent examples of the change in sign of $a_{0}$ are provided by molecular hydrogen (here, $a_{0}$ is positive for the liquid and solid phases, whereas it is negative for the gas [22]) and argon, where the signs of $a_{0}$ are different for the liquid and solid phases $[14,23,24]$. Thus, dependence $a_{0}\left(n_{g}\right)$ is expected for all inert gases, although information on this dependence is scarce. The aim of the present paper is:

1) to try to understand the reasons for the disagreement between experimental data and the optical approximation. There are no physical arguments which could improve the situation in this respect. Under these conditions the applicability of the «optical» approximation has to be checked. We have no fill this gap and to analyze the effect of gas atoms on the electron energy in the states whose localization length is comparable or even less than the interatomic distance;

2) to advance beyond the «optical» approximation where the situation seems to be more understandable. Here it is possible to explain the experimentally observed behavior of $W\left(n_{g}\right)$ including the deviations from the «optical» approximation predictions.

The problem we wish to consider is to determine the energy $W\left(n_{g}\right)$ with the maximum use of information on the polarization interaction of the electron with surrounding atoms

$$
V_{0}(r)=\left\{\begin{array}{cc}
V_{0}>0, & r \leq a, \\
-\alpha e^{2} / 2 r^{4}, & r>a,
\end{array}\right.
$$

including a qualitatively correct passage to the limit of zero gas density. Here $\alpha$ is the atom polarizability and $a$ is the efficient «atom radius» which is considered to be an adjustable parameter.

Actually, the problem consists not only in the systematic treatment of $V_{0}(r)$. Atomic data show (e.g., see Ref. 25), that individual He-atoms exhibiting the lowest among inert gases energy $V_{0}(r)$ (3) form negative ions while all other inert gases are unable to capture a free electron. Therefore the reasonable one-electron formalism has to explain such a paradox. Our original «know how» is mainly concentrated around this place and it could be reduced to the following statement. The existence (or absence) of the bound eigen states in the potential of Eq. (3) is sensitive not only to the atom polarizability $\alpha$, but also to the type of boundary conditions for the wave function imposed at the radius $a$. The proof of this statement is presented in Ref. 26. In particular, the one-electron eigen problem in the potential (3) with the boundary condition for wave function at the infinity $\phi(r \rightarrow \infty)=0$ supplemented by the condition

$$
\phi^{\prime}(a)=0
$$

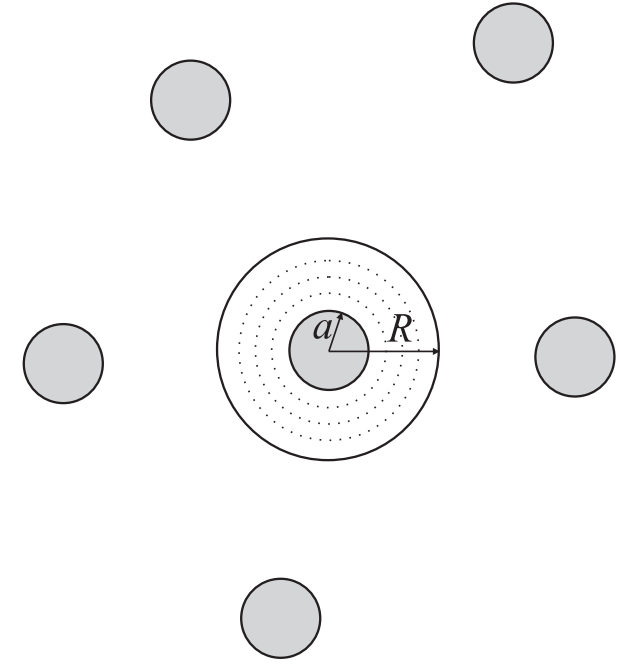

Fig. 1. «Spherically symmetric Wigner-Seitz cell» for an electron in gaseous medium.

has an eigenstate with negative energy, while electron localization does not occur if the boundary condition

$$
\phi(a)=0
$$

are adopted.

The simplest model catching the relevant physics considers a single atom placed at the center of a sphere with the radius $R$ (equal to half the average interparticle distance $n_{g}^{-1 / 3}$ ) interacting via the polarization potential with the electron whose energy is found by solving the Schrödinger equation with the appropriate boundary conditions: first of all, at $r=R$ the wave function radial derivative $\phi^{\prime}(r)$ is zero (a clear analogy with the Wigner-Seitz method in condensed matter physics, the schematic picture of such a cell is presented in Fig. 1); second, at the efficient «atom radius» $r=a \sim a_{B}$ the wave function can satisfy the boundary condition of either Eqs. (4) or (5) which take into account the individual properties of dif-

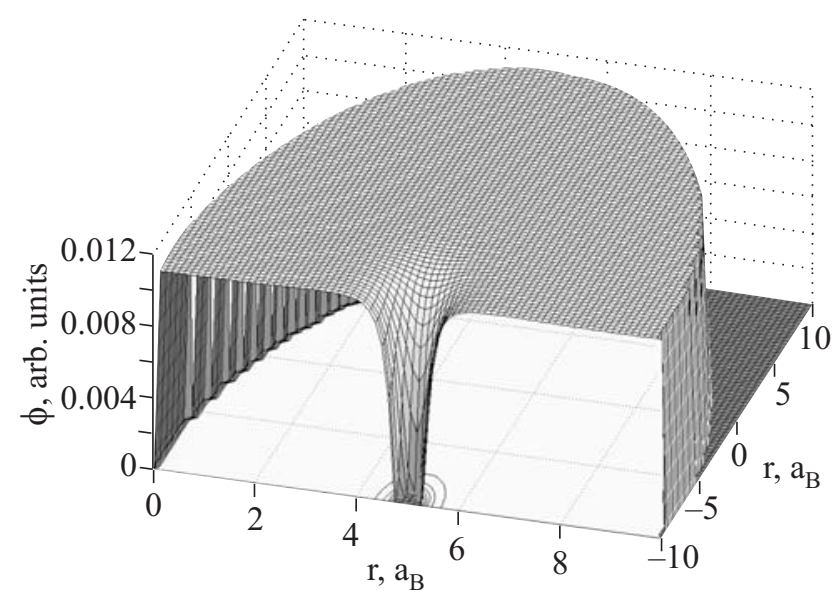

Fig. 2. Electron wave function for the boundary conditions $\phi(r=a)=0, \phi^{\prime}(r=R)=0$. 


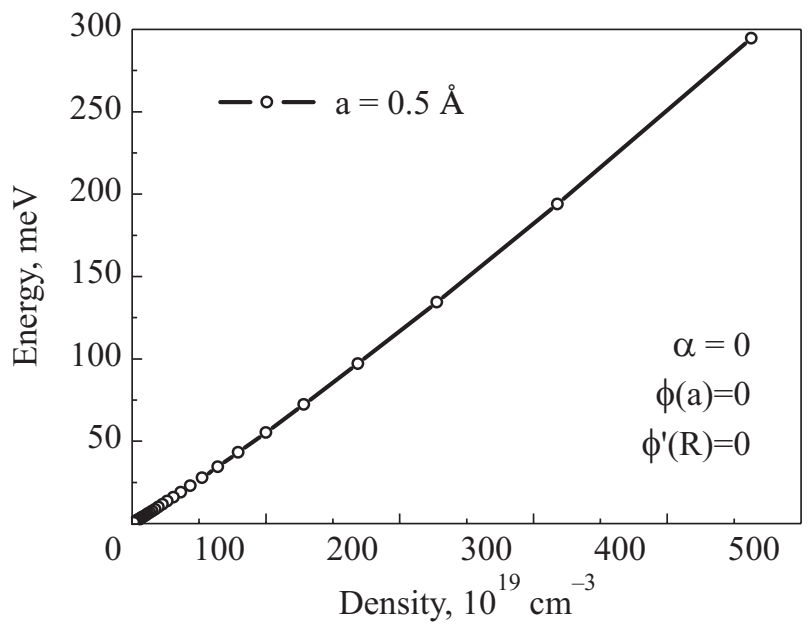

Fig. 3. Minimal energy $W$ as a function of gas density for the case of $\alpha=0$.

ferent inert gases. Having solved the appropriate single-electron Schrödinger equation with the boundary conditions (4) or (5), we should check the desirable consistency between the predictions of the analytical «optical» approximation and numerical results obtained for the extreme case of $\alpha \rightarrow 0$. Here $W\left(n_{g}\right)$ should vary linearly with $n_{g}$ :

$$
\left.W\left(n_{g}\right)\right|_{\alpha \rightarrow 0} \propto n_{g} .
$$

Figure 2 shows these scenarios for $\phi(r)$ satisfying the boundary conditions of Eq. (5). Figure 3 demonstrates the predictable linear behavior (6) of $W\left(n_{g}\right)$. After performing the test of Eq. (6) one can be sure that the non-linear variations of $W\left(n_{g}\right)$ with $n_{g}$ for $\alpha \neq 0$, which are indeed observed in our numerical results, actually reflect the real situation beyond the «optical» approximation.

Then it is possible to build the minimal electron energy $W\left(n_{g}\right)$ in the cell presented in Fig. 1 and compare these

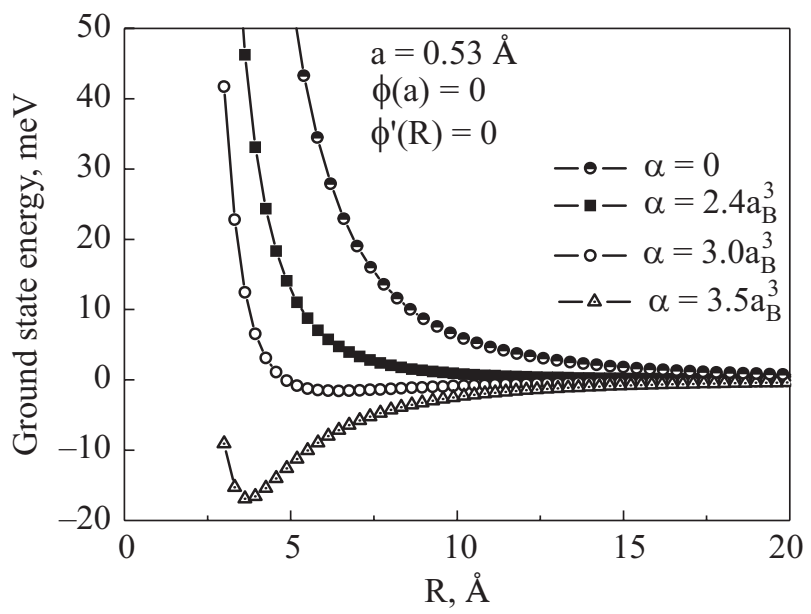

Fig. 4. Ground state energy of electron in a «spherically symmetric Wigner-Seitz cell» versus the spherical cell radius $R$ for different values of atom polarizability.

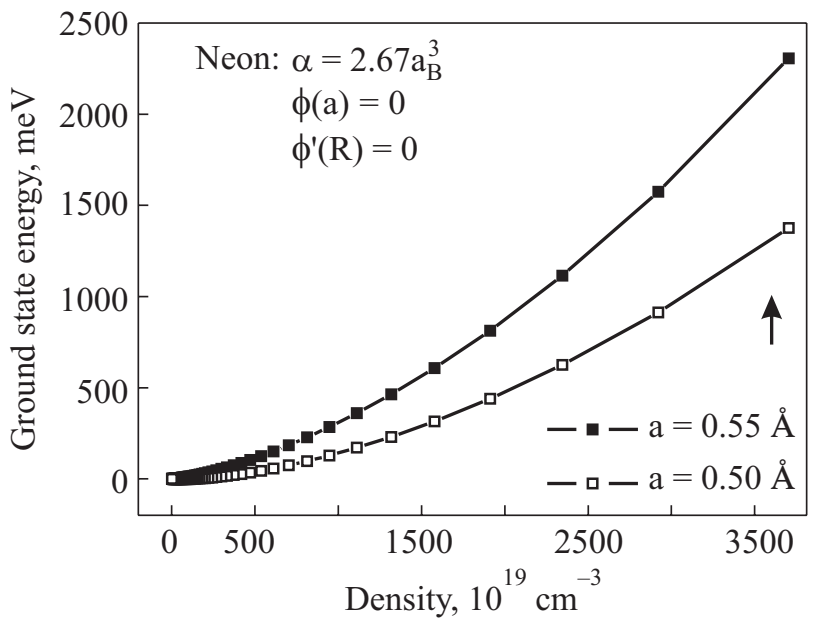

Fig. 5. Energy $W_{\mathrm{Ne}}\left(n_{g}\right)$ for Ne with two different radii $a$. The value $a=0.50 \AA$, for which the energy $W_{\mathrm{Ne}}$ reaches a value of about $1.05 \mathrm{eV}$ [27] for the liquid-phase Ne density, is favorable. This position on the horizontal axis is marked by the arrow.

results with the optical approximation (1). Such a comparison of $W\left(n_{g}\right)$ with $W_{0}\left(n_{g}\right)$ given by Eq. (1) should provide an estimate of the gas density dependence of the effective scattering length $a_{0}$ for small and intermediate values of $n_{g}$ and the unification of available individual data on the $a_{0}\left(n_{g}\right)$ behavior. It helps to find out how universal the optical approximation (1) with $a_{0}\left(n_{g}\right)=$ const is actually (see the results obtained by carrying out this program for Ne presented in Figs. 3 and 4).

There are also even more spectacular results. Let us consider the situation with the boundary conditions given by Eq. (4) which are suitable for all noble gases except for $\mathrm{He}$, and let the atomic polarizability $\alpha$ change. Calculations reveal that by varying $\alpha$ it is possible to obtain the $W\left(n_{g}\right)$ curves which are either monotonously decreasing positive functions of $R=n_{g}^{-1 / 3}$ (for small $\alpha$, like that of

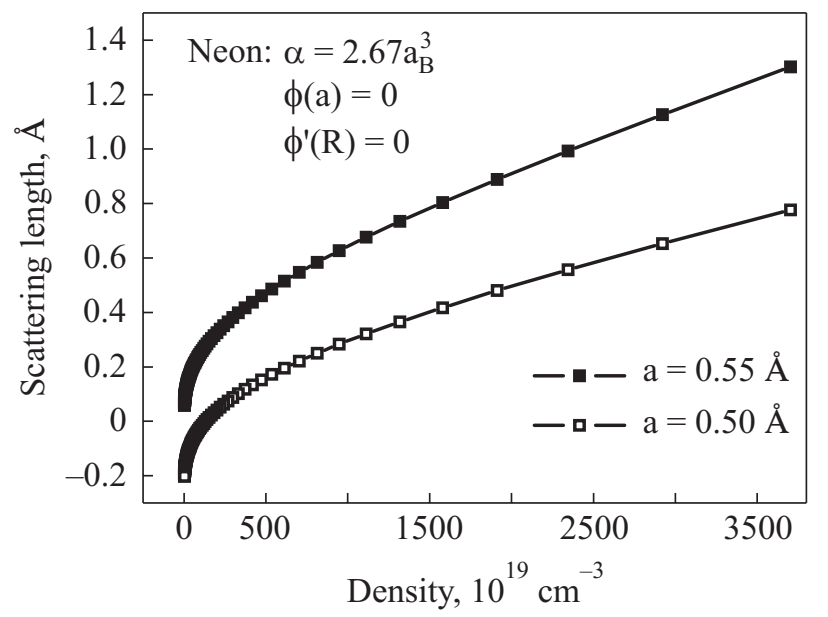

Fig. 6. Electron scattering length on $\mathrm{Ne}$ atoms derived from the data presented in Fig. 5 for $W_{\mathrm{Ne}}\left(n_{g}\right)$. 


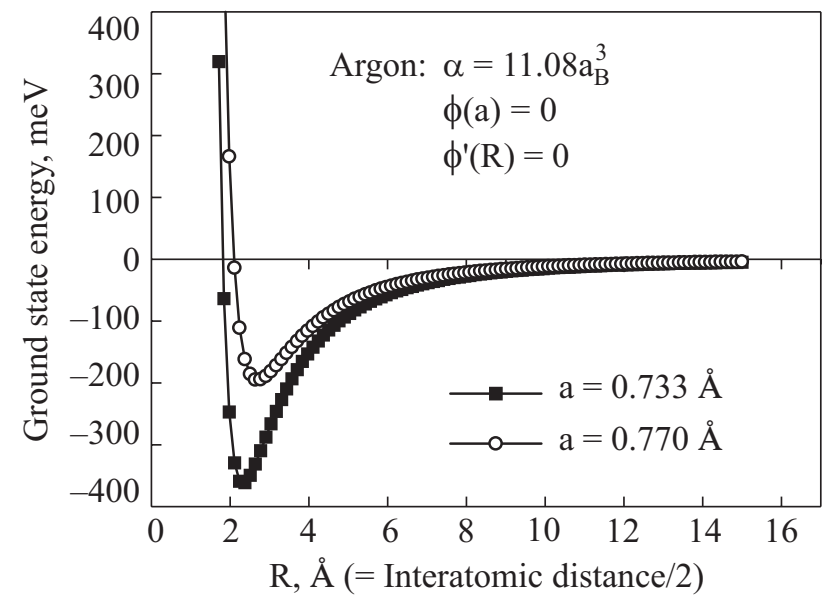

Fig. 7. Ground state energy $W\left(n_{g}\right)$ of electron in Ar versus the spherical cell radius $R$. The two curves show how sensitive the model is to the «atom core» radius $a$.

neon) or exhibit a negative-valued minimum (for sufficiently large $\alpha$ ), as shown in Fig. 5. As a result the way is open to explain the reasons for the above indicated sign variations in $W\left(n_{g}\right)$ both for $\mathrm{H}_{2}$ and argon.

The results obtained by performing this program for $\mathrm{Ne}$ are presented in Figs. 5 and 6. The alternative scenario where $\alpha>\alpha_{\text {crit }}$ and the parameters pertaining to $\mathrm{Ar}$ is shown in Figs. 7 and 8 . The negative part of this energy corresponds to attraction between the electron and liquid argon while the positive one at the liquid-solid transition is responsible for the bubble formation in solid argon.

This work was supported by visitor scientist program of Max Planck Institute for Physics of Complex Systems at Dresden, Germany, the Russian Foundation for Basic Research grant \#06-02-17121 and the Program «Physics of Condensed Matter» of the Presidium of Russian Academy of Sciences.

1. E. Fermi, Nuovo Cim. 11, 157 (1934).

2. H. Margenau and W. Watson, Rev. Mod. Phys. 8, 22 (1936).

3. L. Foldy, Phys. Rev. 67, 107 (1945).

4. B. Burdick, Phys. Rev. Lett. 14, 11 (1965).

5. J. Jortner, N. Kestner, S. Rice, and M. Cohen, J. Chem. Phys. 4, 26143 (1965).

6. B. Springet, J. Jortner, and M. Cohen, J. Chem. Phys. 48, 2720 (1968).

7. L. Tankersley, J. Low Temp. Phys. 11, 451 (1973).

8. A. Fetter, in: The Physics of Liquid and Solid Helium, K. Berreman and J. Ketterson (eds.), Wiley, New York (1976).

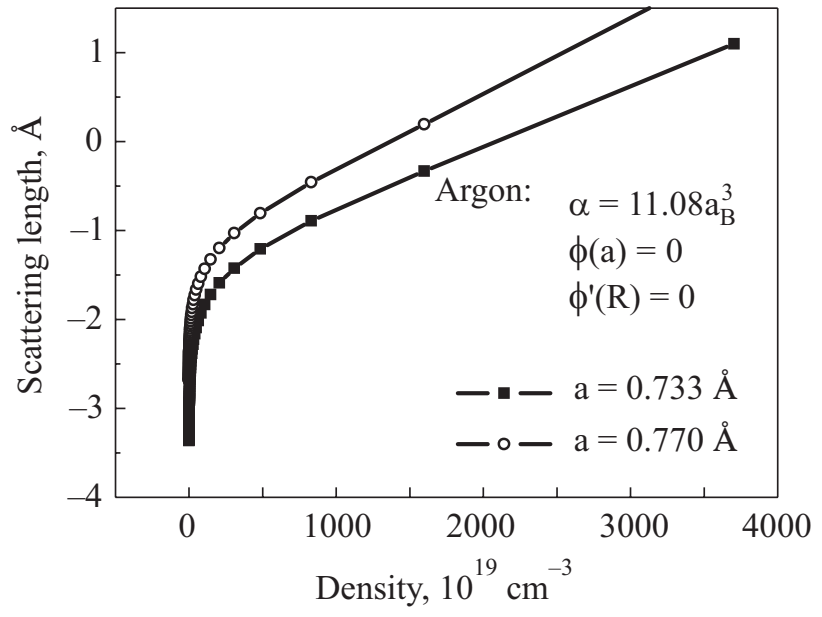

Fig. 8. Electron scattering length on Ar atoms derived from the data presented in Fig. 7 for $W_{\mathrm{Ar}}\left(n_{g}\right)$.

9. E. Bashkin, Zh. Eksp. Teor. Fiz. 82, 1868 (1982) [Sov. Phys. JETP 55, 1076 (1982)].

10. C. Kuper, Phys. Rev. 122, 1007 (1959).

11. J. Levin and T. Sanders, Phys. Rev. 154, 138 (1967).

12. V. Shikin, Usp. Fiz. Nauk 121, 457 (1977) [Sov. Phys. Usp. 20, 226 (1977)].

13. V. Shikin, Pis'ma ZhETF 80, 472 (2004).

14. T. O'Malley, Phys. Rev. 130, 1020 (1963).

15. A. Khrapak and I. Yakubov, Electrons in Dense Gases and Plasma, Nauka, Moscow (1981) [in Russian].

16. B. Smirnov and M. Chibisov, Zh. Eksp. Teor. Fiz. 49, 841 (1965) [Sov. Phys. JETP 22, 585 (1966)].

17. A. Rivier and D. Sweetman, Phys. Rev. Lett. 5, 560 (1966).

18. V. Oparin, R. Il'in, I. Serenkov, E. Solov'ev, and N. Fedorenko, Pis'ma Zh. Eksp. Teor. Fiz. 12, 237 (1970) [JETP Lett. 12, 162 (1970)].

19. M. Woolf and G. Reifield, Phys. Rev. Lett. 1, 2355 (1965).

20. W. Schoepe and G. Reifield, Phys. Rev. A7, 2111 (1973).

21. J. Broomall, W. Johnson, and D. Onn, Phys. Rev. B14, 2819 (1976).

22. V. Zav'yalov and I. Smol'yaninov, Zh. Eksp. Teor. Fiz. 92, 339 (1987) [Sov. Phys. JETP 65, 194 (1987)].

23. J. Lekner, Phys. Rev. 158, 130 (1967).

24. J. Jortner and A. Gaathon, Can. J. Chem. 55, 1801 (1977).

25. B.M. Smirnov, Negative Ions, Atomizdat, Moscow (1978); McGraw-Hill, New York (1982).

26. S. Nazin and V. Shikin, Pis'ma ZhETF 82, 255 (2005).

27. W. Tauchert, H. Jungblut, and W. Schmidt, Can. J. Chem. 55, 1860 (1977).

28. P. Platzman and M. Dikman, Science 284, 1967 (1999).

29. E. Collin, W. Bailey, P. Fozooni, P.G. Frayne, P. Glasson, K. Harrabi, M.J. Lea, and G. Papageorgiou, Phys. Rev. Lett. 89, 245301 (2002). 